\title{
INTEGRALLY CLOSED SUBRINGS OF AN INTEGRAL DOMAIN
}

\author{
BY \\ ROBERT GILMER AND JOE MOTT( $\left.{ }^{1}\right)$
}

\begin{abstract}
Let $D$ be an integral domain with identity having quotient field $K$. This paper gives necessary and sufficient conditions on $D$ in order that each integrally closed subring of $D$ should belong to some subclass of the class of integrally closed domains; some of the subclasses considered are the completely integrally closed domains, Prüfer domains, and Dedekind domains.
\end{abstract}

1. The class of integrally closed domains contains several classes of domains which are of fundamental importance in commutative algebra. Unique factorization domains, Krull domains, domains of finite character, Prüfer domains, completely integrally closed domains, Dedekind domains, and principal ideal domains are examples of such subclasses of the class of integrally closed domains. This paper considers the problems of determining, conversely, necessary and sufficient conditions on an integral domain with identity in order that each of its integrally closed subrings should belong to some subclass of the class of integrally closed domains. An example of a typical result might be Theorem 2.3: If $J$ is an integral domain with identity having quotient field $K$, then conditions (1) and (2) are equivalent. (1) Each integrally closed subring of $J$ is completely integrally closed. (2) Either $J$ has characteristic 0 and $K$ is algebraic over the field of rational numbers or $J$ has characteristic $p \neq 0$ and $K$ has transcendence degree at most one over its prime subfield. If $J$ is integrally closed, then conditions (1) and (2) are equivalent to: (3) Each integrally closed subring of $J$ with quotient field $K$ is completely integrally closed.

In considering characterizations of integral domains with identity for which every integrally closed subring is Dedekind or almost Dedekind ( $\$ 3)$, we are led to use some results of $\mathrm{W}$. Krull to prove Theorem 4.1, which establishes the existence of, as well as a method for constructing, a field with certain specified valuations. We then use this theorem to construct an example of an infinite separable algebraic extension field $K$ of $\Pi_{p}(X)$ such that the integral closure $J$ of $\Pi_{p}[X]$ in $K$

Received by the editors April 7, 1970.

AMS 1969 subject classifications. Primary 1315, 1350; Secondary 1320.

Key words and phrases. Integrally closed domain, completely integrally closed, Prüfer domain, Krull domain, Dedekind domain, almost Dedekind domain.

( $\left.{ }^{1}\right)$ During the writing of this paper, the authors received support under National Science Foundation Grant GP-8424. 
has the property that each integrally closed subring of $J$ is Dedekind, while not every integrally closed subring of $K$ is Dedekind.

2. The case of completely integrally closed subrings. If $D$ is an integral domain with quotient field $K$, an element $x$ in $K$ is said to be almost integral over $D$ if each power of $x$ belongs to some finite $D$-submodule of $K$; this is equivalent to the condition that for some nonzero element $d$ of $D, d x^{n} \in D$ for each positive integer $n$. $D$ is said to be completely integrally closed (c.i.c.) if each element of $K$ almost integral over $D$ is in $D$. Completely integrally closed domains are characterized as those integral domains with identity for which the monoid of divisor classes is a group [2, p. 5]. Any completely integrally closed domain is integrally closed, but the converse fails; in fact, a nontrivial valuation ring is c.i.c. if and only if it has rank $1[13$, p. 170].

We begin by seeking to characterize those integral domains $D$ with identity for which every integrally closed subring is c.i.c. Such a characterization will be basic to further development since unique factorization domains, Krull domains, and Dedekind domains, for example, are representable as intersections of families of rank one valuation rings, and are therefore c.i.c.

THEOREM 2.1. Let $J$ be an integrally closed domain which is not a field. There exists a subring $D$ of $J\left[\left\{X_{\lambda}\right\}\right]$ with these two properties:

(1) $D$ and $J\left[\left\{X_{\lambda}\right\}\right]$ have the same quotient field.

(2) $D$ is integrally closed, but not c.i.c.

Proof. Let $M$ be a maximal ideal of $J$ and set $D=J+M\left[\left\{X_{\lambda}\right\}\right]$. We show that $D$ satisfies (1) and (2). $J\left[\left\{X_{\lambda}\right\}\right]$ and $M\left[\left\{X_{\lambda}\right\}\right]$ have the same quotient field, for $M\left[\left\{X_{\lambda}\right\}\right]$ is a nonzero ideal of $J\left[\left\{X_{\lambda}\right\}\right]$; hence $D$ and $J\left[\left\{X_{\lambda}\right\}\right]$ have the same quotient field. Since $J\left[\left\{X_{\lambda}\right\}\right]$ is integrally closed, (2) holds for $D$ if $D$ is integrally closed in $J\left[\left\{X_{\lambda}\right\}\right]$. But $D$ is integrally closed in $J\left[\left\{X_{\lambda}\right\}\right]$ because $M\left[\left\{X_{\lambda}\right\}\right]$ is a common ideal of $D$ and $J\left[\left\{X_{\lambda}\right\}\right]$ and $D / M\left[\left\{X_{\lambda}\right\}\right] \simeq J / M$ is integrally closed in $J\left[\left\{X_{\lambda}\right\}\right] / M\left[\left\{X_{\lambda}\right\}\right]$ $\simeq(J / M)\left[\left\{X_{\lambda}\right\}\right][17$, p. 34$]$.

REMARK. If $A$ is an ideal of a commutative ring $R$ and if $\left\{X_{\lambda}\right\}$ is a set of indeterminates over $R$, it can be shown in general that $R+(\sqrt{ } A)\left[\left\{X_{\lambda}\right\}\right]$ is the integral closure of $R+A\left[\left\{X_{\lambda}\right\}\right]$ in $R\left[\left\{X_{\lambda}\right\}\right]$. Hence if $R$ is an integrally closed domain, then $R+A\left[\left\{X_{\lambda}\right\}\right]$ is integrally closed if and only if $A=\sqrt{ } A$.

In the remainder of this paper, we use $Z$ to denote the ring of integers, $Q$ to denote the field of rational numbers, and $\Pi_{p}$ to denote the finite field with $p$ elements.

THEOREM 2.2. Let $J$ be an integral domain with identity having quotient field $K$ and suppose that each integrally closed subring of $J$ is c.i.c. If $J$ has characteristic 0 , then $K / Q$ is algebraic. If $J$ has characteristic $p \neq 0$, then the transcendence degree of $K$ over $\Pi_{p}\left(\operatorname{tr} . \mathrm{d} . K / \Pi_{p}\right)$ is at most 1 . If $J$ is integrally closed, then the preceding sentence is valid under the weaker assumption that each integrally closed subring of $J$ with quotient field $K$ is c.i.c. 
Proof. If $J$ has characteristic 0 and if $K / Q$ is not algebraic, then we can choose an element $X$ in $J$ transcendental over $Q$. Then by Theorem $2.1 Z[X]$, and hence $J$, contains an integrally closed subring which is not c.i.c., contrary to hypothesis. It follows that $K / Q$ is algebraic if $J$ has characteristic 0 . Similarly, Theorem 2.1 shows that a polynomial ring in two indeterminates over $\Pi_{p}$ contains a subring which is integrally closed but not c.i.c. Consequently, if $J$ has characteristic $p \neq 0$, then tr.d. $K / \Pi_{p} \leqq 1$.

If $J$ is integrally closed, we consider the case when $J$ has characteristic $p \neq 0$; the resolution of the case when $J$ has characteristic 0 is similar. Thus if tr.d. $K / \Pi_{p}>1$, then we can choose a transcendence basis $B$ for $K / \Pi_{p}$ as a subset of $J$ [21, p. 99]. Then Theorem 2.1 shows that there is an integrally closed subring $D$ of $\Pi_{p}[B]$ with quotient field $\Pi_{p}(B)$ such that $D$ is not c.i.c. Since $K / \Pi_{p}(B)$ is algebraic, the integral closure $D^{\prime}$ of $D$ in $K$ has quotient field $K$. Further, $D^{\prime} \subseteq J$ since $D \subseteq J$ and $J$ is integrally closed. Finally, $D^{\prime} \cap \Pi_{p}(B)=D$ so that $D^{\prime}$ is not c.i.c. since each element of $\Pi_{p}(B)-D$ which is almost integral over $D$ is also an element of $K-D^{\prime}$ which is almost integral over $D^{\prime}$. It follows that if $J$ has characteristic $p \neq 0$ and if each integrally closed subring of $J$ with quotient field $K$ is c.i.c., then tr.d. $K / \Pi_{p} \leqq 1$.

THEOREM 2.3. If $J$ is an integral domain with identity having quotient field $K$, then these conditions are equivalent.

(1) Each integrally closed subring of $J$ is c.i.c.

(2) Either $J$ has characteristic 0 and $K / Q$ is algebraic or $J$ has characteristic $p \neq 0$ and tr.d. $K / \Pi_{p} \leqq 1$.

If $J$ is integrally closed, then (1) and (2) are equivalent to

(3) Each integrally closed subring of $J$ with quotient field $K$ is c.i.c.

Proof. (1) $\rightarrow$ (2). Theorem 2.2 .

$(2) \rightarrow(1)$. If (2) holds, then each subring of $K$ has dimension at most one [7, Theorem 5], and hence each valuation subring of $K$ is c.i.c. Since any integrally closed subring $D$ of $K$ is an intersection of valuation subrings of $K$, it follows that any such $D$ is c.i.c. In particular, (2) holds.

If $J$ is integrally closed, it is obvious that (1) implies (3), and the converse follows from Theorem 2.2.

Remarks. Given an integral domain $D$ with identity, $D$ need not contain an integrally closed subring having the same quotient field which $D$ has. This is true, for example, if $D$ has characteristic 0 , is integral over $Z$, and is not integrally closed. Hence condition (3) of Theorem 2.3 does not imply conditions (1) and (2) for an arbitrary integral domain with identity.

Using results from [7] and $[9, \S 16]$, it follows that for a field $J$, conditions (1)-(3) of Theorem 2.3 are equivalent to each of these conditions: (4) Each valuation ring on $J$ has rank $\leqq 1$. (5) Each valuation subring of $J$ has rank $\leqq 1$. (6) Each subring of $J$ with identity has dimension $\leqq 1$. 
3. Some domains of classical ideal theory. Among the subclasses of the class of integrally closed domains are the classes of Prüfer domains, Krull domains, almost Dedekind domains $\left({ }^{2}\right)$, and Dedekind domains. In fact, these last three classes are contained in the class of completely integrally closed domains. In this section we seek conditions on a domain with identity in order that each of its integrally closed subrings belong to one of the four subclasses previously mentioned. It is interesting to note that in considering the condition that each integrally closed subring be either Dedekind or Krull, we are led to a universal property on subrings that is independent of integral closure, namely, the Noetherian condition; see Theorem 3.4 in this connection.

Our first considerations are of the case of Prüfer domains. Theorem 3.1 uses this terminology: We say that an integral domain $D$ with identity satisfies the $Q R$ property if each overring of $D$ is a quotient ring of $D$. Domains with the $Q R$ property were first considered in [10] and in [5]; in [18], Pendleton proved that $D$ has the $Q R$-property if and only if $D$ is Prüfer and the radical of each finitely generated ideal of $D$ is the radical of a principal ideal of $D$.

THEOREM 3.1. Suppose that $J$ is an integral domain with identity having quotient field $K$. The following conditions are equivalent.

(1) Each integrally closed subring of $J$ is a Prüfer domain.

(2) Either $K$ has characteristic 0 and $K / Q$ is algebraic or $K$ has characteristic $p \neq 0$ and tr.d. $K / \Pi_{p} \leqq 1$.

(3) Each integrally closed subring of $J$ is c.i.c.

(4) Each integrally closed subring of $K$ has the QR-property.

(5) Each integrally closed subring of $J$ has the QR-property.

Proof. (1) $\rightarrow(2)$. It is known that if $D$ is a Prüfer domain and a Krull domain, then $D$ is Dedekind and therefore has dimension at most $1[9$, p. 525]. $Z[X]$ and $\Pi_{p}[X, Y]$ are two-dimensional UFD's, and hence they are Krull domains. We conclude, therefore, that if (1) holds, then (2) also holds.

(2) $\leftrightarrow$ (3). Theorem 2.3 .

$(2) \rightarrow(4)$. [5, p. 202].

Clearly (4) implies (5), and (5) implies (1) since a domain with the $Q R$-property is Prüfer [10, p. 99].

THEOREM 3.2. Let $J$ be an integral domain with identity having quotient field $K$. These conditions are equivalent.

(1) Each integrally closed subring of $K$ is almost Dedekind.

(2) Each integrally closed subring of $K$ with quotient field $K$ is almost Dedekind.

$\left({ }^{2}\right)$ An almost Dedekind domain is an integral domain $D$ with identity such that $D_{M}$ is a Noetherian valuation ring for each maximal ideal $M$ of $D$; see [8] or $[9, \S 29]$. 
(3) Each valuation ring on $K$ is Noetherian.

(4) Either $K$ has characteristic $0, K / Q$ is algebraic, and the integral closure of $Z$ in $K$ is almost Dedekind, or $K$ has characteristic $p \neq 0$, tr.d. $K / \Pi_{p} \leqq 1$, and in the case where tr.d. $K / \Pi_{p}=1$, there is a transcendence basis $X$ for $K / \Pi_{p}$ such that the integral closures $D_{1}$ and $D_{2}$ of $\Pi_{p}[X]$ and $\Pi_{p}[1 / X]$, respectively, in $K$ are almost Dedekind.

If $K$ has characteristic 0 and if $J$ is integrally closed, then (1) is equitalent to

(5) Each integrally closed subring of $J$ with quotient field $K$ is almost Dedekind.

Proof. (1) $\rightarrow$ (2) is clear, and (2) implies (3) since an almost Dedekind valuation ring must be Noetherian.

(3) $\rightarrow$ (4). If $K$ has characteristic 0 , then $K / Q$ is algebraic by Theorem 2.3. If $Z^{\prime}$ is the integral closure of $Z$ in $K$, then $Z^{\prime}$ is a one-dimensional Prüfer domain by Theorem 3.1, so that $\left(Z^{\prime}\right)_{M}$ is a Noetherian valuation ring on $K$ for each maximal ideal $M$ of $Z^{\prime}$. It follows that $Z^{\prime}$ is almost Dedekind. The proof in the case when $K$ has characteristic $p \neq 0$ is entirely analogous to the case when $K$ has characteristic 0 , and in fact it is true that if tr.d. $K / \Pi_{p}=1$, then for any transcendence basis $Y$ for $K / \Pi_{p}$, the integral closure of $\Pi_{p}[Y]$ in $K$ is almost Dedekind.

(4) $\rightarrow(1)$. We first consider the case when $K$ has characteristic 0 . If $D$ is an integrally closed subring of $K$, then $D^{\prime}$, the integral closure of $D$ in $K$, has quotient field $K$. Further, if $D^{\prime}$ is almost Dedekind, then so is $D[12$, p. 750$]$. Hence, we can assume that $D$ has quotient field $K$. By Theorem 3.1, $D$ is a one-dimensional Prüfer domain (we exclude the case when $D=K$ ). Hence $D_{M}$ is a rank one valuation ring for each maximal ideal $M$ of $D$. If $K$ has characteristic 0 , then $D_{M}$ is an overring of the integral closure $Z^{\prime}$ of $Z$ in $K$; if $K$ has characteristic $p \neq 0$, then $D_{M}$ is an overring of either $D_{1}$ or $D_{2}$. In any case, $D_{M}$ is rank one discrete since $Z^{\prime}, D_{1}$, and $D_{2}$ are assumed to be almost Dedekind and any overring of an almost Dedekind domain is almost Dedekind [8, p. 816]. Hence, $D$ is almost Dedekind.

That (1) implies (5) is patent. If (5) holds, then Theorem 3.1 shows that $K / Q$ is algebraic. Further, the integral closure $Z^{\prime}$ of $Z$ in $K$ is a subring of $J$ with quotient field $K$, and is therefore almost Dedekind.

COROLLARY 3.3. If $J$ is an integrally closed domain with quotient field $K$ such that each integrally closed subring of $J$ with quotient field $K$ is almost Dedekind, then each integrally closed subring of $\mathrm{J}$ is almost Dedekind.

Proof. If $J$ has characteristic 0 , then Theorem 3.2 yields Corollary 3.3. If $J$ has characteristic $p \neq 0$, then by Theorem 3.1 , tr.d. $K / \Pi_{p} \leqq 1$. If $D$ is an integrally closed subring of $J$, then either $D$ is a field or the transcendence degree of the quotient field of $D$ over $\Pi_{p}$ is 1 . In this latter case, the integral closure $D^{\prime}$ of $D$ in $K$ is an integrally closed subring of $J$ with quotient field $K$. Thus $D^{\prime}$, and hence $D$, is almost Dedekind.

We shall show, in Example 4.2, that an integrally closed domain $J$ of characteristic $p \neq 0$ can have the property that each integrally closed subring of $J$ is a 
Dedekind domain, while $K$, the quotient field of $J$, admits a rank one nondiscrete valuation ring.

THEOREM 3.4. Suppose that $J$ is an integrally closed domain with quotient field $K$ and that $\mathscr{S}$ is the family of integrally closed subrings of $J$ with quotient field $K$. These conditions are equivalent.

(1) Each $D$ in $\mathscr{S}$ is Dedekind.

(2) Each $D$ in $\mathscr{S}$ is Noetherian.

(3) Each $D$ in $\mathscr{S}$ is a Krull domain.

(4) Each integrally closed subring of $J$ is a Dedekind domain.

If $J$ has characteristic 0 , then conditions (1)-(4) are equivalent to each of the following conditions.

(5) The integral closure of $Z$ in $K$ is Dedekind.

(6) Each integrally closed subring of $K$ is Dedekind.

Proof. The proof of the equivalence of (1) and (4)-(6) proceeds as in the proof of Theorem 3.2, once we recall the following result due to Heinzer [12, p. 750]: If $D_{0}$ is an integrally closed domain with quotient field $K_{0}$, if $K_{1}$ is an algebraic extension field of $K_{0}$, and if $D_{1}$ is the integral closure of $D_{0}$ in $K_{1}$, then $D_{0}$ is Dedekind if $D_{1}$ is Dedekind.

We establish the equivalence of (1)-(3). It is clear that (1) implies (2) and (2) implies (3) since a Noetherian integrally closed domain is a Krull domain. If (3) holds, then each $D$ in $\mathscr{S}$ is c.i.c. since a Krull domain is c.i.c. Then Theorems 2.3 and 3.1 show that each $D$ in $S$ has dimension $\leqq 1$. Since a Krull domain of dimension $\leqq 1$ is a Dedekind domain [22, p. 84], it follows that (1) holds.

THEOREM 3.5. Suppose that $K$ is a field of characteristic $p \neq 0$ of transcendence degree 1 over $\Pi_{p}$. These conditions are equivalent.

(1) Each integrally closed subring of $K$ is Dedekind.

(2) There is a transcendence basis $X$ for $K / \Pi_{p}$ such that the integral closures $D_{1}$ and $D_{2}$ of $\Pi_{p}[X]$ and $\Pi_{p}[1 / X]$, respectively, in $K$ are Dedekind domains.

Proof. We prove that (2) implies (1). By Theorem 3.4, it suffices to show that if $D$ is an integrally closed subring of $K$ with quotient field $K$, then $D$ is a Dedekind domain. Further, $D$ is almost Dedekind by Theorem 3.2. If $V$ is a valuation ring on $K$, then $V$ contains $X$ or $1 / X$, and hence $V$ contains $D_{1}$ or $D_{2}$. It follows that $\mathscr{F}_{1} \cup \mathscr{F}_{2}$ is the family of nontrivial valuation rings on $K$, where $\mathscr{F}_{i}$ is the family of nontrivial valuation overrings of $D_{i}$. Because $D_{1}$ and $D_{2}$ are Dedekind domains, the family $\mathscr{F}_{1} \cup \mathscr{F}_{2}$ has finite character. $D$ is the intersection of a subfamily of $\mathscr{F}_{1} \cup \mathscr{F}_{2}$, and hence $D$ is a Krull domain. Consequently, $D$ is Dedekind.

REMARK. Let $F$ be an algebraic field extension of $\Pi_{p}$. Then $F[X]$ is the integral closure of $\Pi_{p}[X]$ in $F(X)$ for any indeterminate $X$ over $F$. By Theorem 3.5, each integrally closed subring of $F(X)$ is a Dedekind domain. But by Theorem 4 of [7], each subring of $F(X)$ is Noetherian if and only if $\left[F: \Pi_{p}\right]$ is finite. 
4. A construction of fields with certain specified valuations. In order to obtain an example of an integrally closed domain $J$ with quotient field $K$ such that each integrally closed subring of $J$ is Dedekind, while $K$ admits a rank one nondiscrete valuation ring, we consider in this section the problem of constructing an extension field $K$ of $\Pi_{p}(X)$ such that the set of valuation rings on $K$ is, in some sense, specified. In this connection, we use the following statement $\left({ }^{*}\right)$, which is a consequence of Lemma 4.2 of [1].

(*) Let $\left\{U_{i}\right\}_{i=1}^{r},\left\{V_{i}\right\}_{i=1}^{s}$, and $\left\{W_{i}\right\}_{i=1}^{t}$ be three finite collections of rank one discrete valuation rings on a field $K$ and suppose that each of these valuation rings has finite residue field. Assume also that there is an infinite collection of rank one discrete valuation rings on $K$. Then there exists a separable quadratic extension field $K(t)$ of $K$ such that each $U_{i}$ is inertial with respect to $K(t)$, each $V_{i}$ ramifies with respect to $K(t)$, and each $W_{i}$ decomposes with respect to $K(t)$.

Our terminology in $\left({ }^{*}\right)$ is as follows. If $V$ is a valuation ring on a field $K$ and if $K(t)$ is a separable quadratic extension field of $K$, then it is known that one of three cases occurs (see [4, p. 1] or [20, p. 43]): (1) $V$ has a unique extension $W$ to $K(t)$; $W$ and $V$ have the same value group, and the residue field of $W$ is a quadratic extension of the residue field of $V$. In this case we say that $V$ is inertial with respect to $K(t)$. (2) $V$ has a unique extension $W$ to $K(t) ; W$ and $V$ have the same residue field, and the value group of $V$ is a subgroup of the value group of $W$ of index 2. Under these conditions we say that $V$ ramifies with respect to $K(t)$. (3) $V$ has two extensions $W_{1}, W_{2}$ to $K(t) ; W_{i}$ has the same value group and the same residue field that $V$ has. This is the case where $V$ decomposes with respect to $K(t)$.

Result $\left({ }^{*}\right)$ is a special case of a much more general result [14] due to Krull, who considered the following question. Suppose that $\left\{V_{i}\right\}_{i=1}^{k}$ is a family of rank one discrete valuation rings on a field $K$, and suppose that $V_{i}$ has value group $G_{i}$ and residue field $\Delta_{i}$ for each $i$. For $1 \leqq i \leqq k$, suppose that a set $\left\{G_{i j}\right\}_{j=1}^{r_{i}}$ of totally ordered abelian groups containing $G_{i}$ as a subgroup of finite index is given, and that a collection $\left\{\Delta_{i j}\right\}_{j=1}^{r_{i}}$ of finite algebraic extension fields of $\Delta_{i}$ is given. Suppose further that $\sum_{j=1}^{r_{i}}\left[\Delta_{i j}: \Delta_{i}\right]\left[G_{i j}: G_{i}\right]=n$ for each $i$ between 1 and $k$. Does there exist an $n$-dimensional separable extension field $L$ of $K$ such that for $1 \leqq i \leqq k, V_{i}$ has $r_{i}$ extensions $V_{i 1}, \ldots, V_{i r_{i}}$ to $L$, where $V_{i j}$ has residue field $\Delta_{i j}$ and value group $G_{i j}$ ? Among the sufficient conditions which Krull gives in order that the answer to this question be affirmative are that either (1) or (2) holds: (1) Some $r_{j}$ is one. (2) There exists a rank one discrete valuation ring $V$ on $K$ distinct from each $V_{i}$.

For a historical development of the above material of Krull, as well as extensions of Krull's results, see [6].

THEOREM 4.1. Suppose that $\mathscr{C}$ is a countably infinite collection of rank one discrete valuation rings on a field $K$, that each element of $\mathscr{C}$ has finite residue field, and that $\mathscr{C}=\bigcup_{i=1}^{6} \mathscr{C}_{i}$ is a partitioning of $\mathscr{C}$ into six subsets, some of which may be empty. There is an infinite separable extension field $L$ of $K$ such that each element of 
$\mathscr{C}_{1}$ has only finitely many extensions to $L$, each of which is discrete; each element of $\mathscr{C}_{2}$ has only finitely many extensions to L, each of which is nondiscrete; each element of $\mathscr{C}_{3}$ has infinitely many extensions to $L$, each of which is discrete; each element of $\mathscr{C}_{4}$ has infinitely many extensions to $L$, all of which are nondiscrete; each element of $\mathscr{C}_{5}$ has only finitely many extensions to $L$, some of which are discrete and some of which are nondiscrete; and each element of $\mathscr{C}_{6}$ has infinitely many extensions to $L$, some of these extensions being discrete, while others are nondiscrete.

Proof. For each $i$, let $\mathscr{C}_{i}=\left\{V_{i j}\right\}_{j=1}^{\infty}$. By $\left(^{*}\right)$, there is a separable quadratic extension field $K_{1}=K\left(t_{1}\right)$ of $K$ such that $V_{11}$ is inertial with respect to $K_{1}, V_{21}$ ramifies with respect to $K_{1}$, while $V_{31}, V_{41}, V_{51}$, and $V_{61}$ decompose with respect to $K_{1}$. (If any $\mathscr{C}_{i}$ is empty, we delete the reference to $V_{i 1}$.) We now reapply $\left({ }^{*}\right)$ to the family of valuations on $K_{1}$ which are extensions to $K_{1}$ of some $V_{i 1}$ or $V_{i 2}$. This is a finite family of rank one discrete valuation rings on $K_{1}$, each having finite residue field, and since $\mathscr{C}$ is infinite, the family of rank one discrete valuation rings on $K_{1}$ is infinite. By $\left({ }^{*}\right)$, we can choose a separable quadratic extension field $K_{2}=K_{1}\left(t_{2}\right)$ $=K\left(t_{1}, t_{2}\right)$ of $K_{1}$ such that these conditions hold: Each extension to $K_{1}$ of $V_{11}$ or $V_{12}$ is inertial with respect to $K_{2}$. Each extension to $K_{1}$ of $V_{21}$ or $V_{22}$ or $V_{41}$ or $V_{42}$ ramifies with respect to $K_{2}$. Each extension to $K_{1}$ of $V_{31}$ or $V_{32}$ or $V_{52}$ or $V_{62}$ decomposes with respect to $K_{2}$. Of the two extensions $V_{51}^{(1)}, V_{52}^{(2)}$ of $V_{51}$ to $K_{1}$ and of the two extensions $V_{61}^{(1)}$ and $V_{62}^{(2)}$ of $V_{61}$ to $K_{1}$, we require that $V_{51}^{(1)}$ be inertial with respect to $K_{2}$, that $V_{51}^{(2)}$ and $V_{61}^{(1)}$ ramify with respect to $K_{2}$, and that $V_{61}^{(2)}$ decompose with respect to $K_{2}$. We now reapply (*) to the family of valuation rings on $K_{2}$ which are extensions to $K_{2}$ of some $V_{i j}$, where $j$ is 1,2 , or 3 . There is a separable quadratic extension field $K_{3}=K_{2}\left(t_{3}\right)=K\left(t_{1}, t_{2}, t_{3}\right)$ of $K_{2}$ such that these conditions hold: Each extension to $K_{2}$ of $V_{11}, V_{12}$, or $V_{13}$ is inertial with respect to $K_{3}$. Each extension to $K_{2}$ of $V_{21}, V_{22}$, or $V_{23}$ ramifies with respect to $K_{3}$. Each extension to $K_{2}$ of any of the valuations $V_{31}, V_{32}, V_{33}, V_{41}, V_{42}, V_{43}, V_{53}$, or $V_{63}$ decomposes with respect to $K_{3}$. We require that the unique extension of $V_{51}^{(1)}$ to $K_{2}$ be inertial with respect to $K_{3}$, that the unique extensions to $K_{2}$ of $V_{51}^{(2)}$ and $V_{61}^{(1)}$ ramify with respect to $K_{3}$, and that each of the extensions to $K_{2}$ of $V_{61}^{(2)}$ decomposes with respect to $K_{3}$. Finally, each of $V_{52}$ and $V_{62}$ have at least two extensions to $K_{2}$ (since each extension to $K_{1}$ of $V_{52}$ or $V_{62}$ decomposes with respect to $K_{2}$ ). Let $V_{j 2}^{(1)}$ be one of the extensions of $V_{j 2}$ to $K_{2}$ for $j=5$ or 6 . We require that $V_{52}^{(1)}$ be inertial with respect to $K_{3}$, that $V_{62}^{(1)}$ ramify with respect to $K_{3}$, that each extension to $K_{2}$ of $V_{52}$ other than $V_{52}^{(1)}$ ramifies with respect to $K_{3}$, and that each extension to $K_{2}$ of $V_{62}$ other than $V_{62}^{(1)}$ decomposes with respect to $K_{3}$.

The scheme of our construction is either apparent by now or it can be readily discerned by constructing a diagram indicating decomposition, inertia, or ramification at each stage. Continuing, we obtain an infinite separable extension field $L=K\left(t_{1}, t_{2}, t_{3}, \ldots\right)$ of $K$ satisfying the requirements of Theorem 4.1. While the verification that the conclusion of Theorem 4.1 holds for the field $L$ is rather long 
and detailed, it is nevertheless routine and follows standard lines of argument concerning extensions of valuations, such as may be found in $[22, \S \$ 11,12]$.

It should be noted that according to the construction outlined in the proof of Theorem 4.1, $V_{11}$ and $V_{21}$ will have unique extensions to $L$. Moreover, of the extensions of a given element $V_{5 i}$ to $L$, only one is discrete, while only one of the extensions of a given element $V_{6 i}$ to $L$ is nondiscrete. The vast possibilities for modifying this particular outcome should be obvious. For example, if $\mathscr{F}_{1}$ is a finite subset of $\mathscr{C}_{1}$, we could require that $L$ be such that each element of $\mathscr{F}_{1}$ has a unique extension to $L$.

EXAMPLE 4.2. We give an example of an infinite separable algebraic extension field $K$ of $\Pi_{p}(X)$ such that the integral closure $J$ of $\Pi_{p}[X]$ in $K$ has the property that each integrally closed subring of $J$ is a Dedekind domain, while $K$ admits a rank one nondiscrete valuation ring. The set of nontrivial valuation rings on $\Pi_{p}(X)$ is $\mathscr{C}_{1} \cup \mathscr{C}_{2}$, where $\mathscr{C}_{1}$ is the family of $p(X)$-adic valuation rings on $\Pi_{p}(X), p(X)$ running over a complete set of nonassociate irreducible polynomials in $\Pi_{p}[X]$, and where $\mathscr{C}_{2}$ contains only the $(1 / X)$-adic valuation ring of $\Pi_{p}[1 / X][22$, p. 39]. Since $\Pi_{p}[X]$ is countable, $\mathscr{C}_{1}$ is also countable, and $\mathscr{C}_{1}$ is infinite-say $\mathscr{C}_{1}=\left\{V_{i}\right\}_{i=1}^{\infty}$. We let $\mathscr{C}_{2}=\left\{V_{0}\right\}$. By Theorem 4.1, there exists an infinite separable extension field $K$ of $\Pi_{p}(X)$ such that each element of $\mathscr{C}_{1}$ has only finitely many extensions to $K$, each of which is rank one discrete, while $V_{0}$ has (by the construction in the proof of Theorem 4.1) a unique extension $W_{0}$ to $K$, where $W_{0}$ is not discrete. It then follows that $J$, the integral closure of $\Pi_{p}[X]$ in $K$ is a Dedekind domain [1, p. 142]. We observe that $J$ is the only integrally closed subring of $J$ with quotient field $K$. Thus if $D$ is an integrally closed subring of $K$ with quotient field $K$, then $D$ is the intersection of its set of valuation overrings. Any valuation ring on $K$ contains $X$ or $1 / X$, and hence contains $J$ or $W_{0}$. Since $J \cap W_{0}$ is the algebraic closure of $\Pi_{p}$ in $K$ [9, Theorem 16.7, p. 212], $J \cap W_{0}$ does not have quotient field $K$. Therefore $D \nsubseteq W_{0}$, and we conclude that $D=J$. We have proved that each integrally closed subring of $J$ with quotient field $K$ is a Dedekind domain; by Theorem 3.4, each integrally closed subring of $J$ is Dedekind.

REMARK. We conjecture that domains of the type occurring in Example 4.2 are essentially the only integrally closed domains $J$ such that every integrally closed subring of $J$ is Dedekind, while not every integrally closed subring of $K$, the quotient field of $J$, is Dedekind. To be more explicit, Theorem 3.4 shows that such a domain $J$ is necessarily of characteristic $p \neq 0$, and the transcendence degree of $K$ over $\Pi_{p}$ is necessarily 1 . Hence if $X \in J, X$ transcendental over $\Pi_{p}$, then the integral closure of $\Pi_{p}[X]$ in $K$ is Dedekind. By Theorem 3.5, the integral closure of $\Pi_{p}[1 / X]$ in $K$ is not Dedekind, and this implies that $V_{0}$, the $(1 / X)$-adic valuation on $\Pi_{p}(X)$, either has infinitely many extensions to $K$ or it has an extension to $K$ which is not discrete. We conjecture that what is, in fact, true is that $V_{0}$ has a unique extension to $K$, and this extension is not discrete. That this condition is sufficient to imply that each integrally closed subring of the integral closure of $\Pi_{p}[X]$ in $K$ is Dedekind 
follows as in the presentation of Example 4.2. To prove our conjecture (that is, to prove that this condition is also necessary), it would suffice to prove the following: If $F$ is a finite algebraic extension field of $\Pi_{p}(X)$ such that $V_{0}$ has more than one extension to $F$, then for any extension $W_{0}$ of $V_{0}$ to $F$, the quotient field of $D \cap W_{0}$ is not algebraic over $\Pi_{p}$; here $D$ denotes the integral closure of $\Pi_{p}[X]$ in $F$.

5. Some further results. The class of integrally closed domains contains several important subclasses other than those which we have already considered. In this section we state some results concerning the characterization problems for certain of these subclasses. Since almost all of the methods of proof of these results have already appeared in $\$ \S 2,3$, no proofs are presented in this section.

THEOREM 5.1. Let $J$ be an integral domain with identity. Each integrally closed subring of $J$ is a PID if and only if each integrally closed subring of $J$ is a UFD.

REMARK. The characterization given in Theorem 5.1 is not very satisfactory. For example, when $J$ is an algebraic number field, then the conditions of Theorem 5.1 are equivalent to the assertion that the class number of each subfield of $J$ is 1 . Even when $[J: Q]$ is finite, no such characterization of such fields is known in algebraic number theory.

The class number of each subfield of $Q(\sqrt{ }-19)$ is 1 , so that each integrally closed subring of $Q(\sqrt{ }-19)$ is a PID, but the integral closure of $Z$ in $Q(\sqrt{ }-19)$ is not a Euclidean domain [15, p. 1144]. Hence, the conditions of Theorem 5.1 do not imply that each integrally closed subring of $J$ is a Euclidean domain. Again, the classification of those finite algebraic number fields such that each integrally closed subring is Euclidean is an open problem in algebraic number theory.

A Krull domain is the classical example of a domain which is defined as a family of valuation rings satisfying certain axioms. Several generalizations of the notion of a Krull domain have appeared in the literature and have proved to be significant. Among these generalizations are the concepts of a domain of finite character, a domain of finite real character, a domain of Krull type, and a generalized Krull domain. (See [19], [16], [11], [9, §35], and [3] for definitions and pertinent results.) We consider these concepts in Theorem 5.2.

THEOREM 5.2. Let $K$ be a field and let $\mathscr{S}$ be the family of integrally closed subrings of $K$ with quotient field $K$. These conditions are equivalent.

(1) Each $D$ in $\mathscr{S}$ is a generalized Krull domain.

(2) Each $D$ in $\mathscr{S}$ is a Prüfer domain of finite real character with dimension at most one.

(3) Each $D$ in $\mathscr{S}$ is a domain of finite real character.

(4) Either $K$ has characteristic $0, K / Q$ is algebraic, and the integral closure of $Z$ in $K$ is a generalized Krull domain, or $K$ has characteristic $p \neq 0$, tr.d. $K / \Pi_{p} \leqq 1$, and in the case when tr.d. $K / \Pi_{p}=1$, then for any transcendence basis $X$ for $K / \Pi_{p}$, the integral closures in $K$ of $\Pi_{p}[X]$ and $\Pi_{p}[1 / X]$ are generalized Krull domains. 
(5) Each $D$ in $\mathscr{S}$ is of Krull type.

(6) Each $D$ in $\mathscr{S}$ is of finite character.

The methods required to establish all the implications of Theorem 5.2 except the implication $(6) \rightarrow(4)$ have already appeared in $\$ \S 2,3$, so we omit the proof of Theorem 5.2. To show that $(6) \rightarrow(4)$ it is sufficient to prove that if $V$ is a nontrivial valuation ring on a field $F$ with residue field $\Delta$ not algebraic over its prime subfield, then there is an integrally closed subring $D$ of $V$ with quotient field $F$ such that $D$ is not of finite character. The result in the preceding sentence can be established by using the construction appearing in Appendix 2 of [9].

\section{REFERENCES}

1. J. Arnold and R. Gilmer, Idempotent ideals and unions of nets and Prüfer domains, J. Sci. Hiroshima Univ. Ser. A-I Math. 31 (1967), 131-145. MR 37 \#2741.

2. N. Bourbaki, Algèbre commutative, Chap. 7, Hermann, Paris, 1965.

3. J. Brewer and J. Mott, Integral domains of finite character, J. Reine Angew. Math. 241 (1970), 34-41.

4. I. S. Cohen and O. Zariski, A fundamental inequality in the theory of extensions of valuations, Illinois J. Math. 1 (1957), 1-8. MR 18, 788.

5. E. D. Davis, Overrings of commutative rings. II. Integrally closed overrings, Trans. Amer. Math. Soc. 110 (1964), 196-212. MR 28 \#111.

6. O. Endler, Endliche separable Körpererweiterungen mit vorgeschriebenen Bewertungsfortsetzungen. I, Abh. Math. Sem. Hamburg 33 (1969), 80-101. MR 39 \#4124.

7. R. Gilmer, Integral domains with Noetherian subrings, Comment. Math. Helv. 45 (1970), $129-134$.

8. - Integral domains which are almost Dedekind, Proc. Amer. Math. Soc. 15 (1964), 813-818. MR 29 \#3489.

9. - Multiplicative ideal theory, Queen's Papers in Pure and Appl. Math., no. 12, Queen's University, Kingston, Ont., 1968. MR 37 \#5198.

10. R. Gilmer and J. Ohm, Integral domains with quotient overrings, Math. Ann. 153 (1964), 97-103. MR 28 \#3051.

11. M. Griffin, Families of finite character and essential valuations, Trans. Amer. Math. Soc. 130 (1968), 75-85. MR 36 \#1423.

12. W. Heinzer, Some properties of integral closure, Proc. Amer. Math. Soc. 18 (1967), 749753. MR 35 \#5429.

13. W. Krull, Allgemeine Bewertungstheorie, J. Reine Angew. Math. 167 (1932), 160-196.

14. — Uber einen Existenzsatz der Bewertungstheorie, Abh. Math. Sem. Univ. Hamburg 23 (1959), 29-35. MR 21 \#3406.

15. T. Motzkin, The Euclidean algorithm, Bull. Amer. Math. Soc. 55 (1949), 1142-1146. MR 11, 311.

16. J. Ohm, Some counterexamples related to integral closure in $D[[X]]$, Trans. Amer. Math. Soc. 122 (1966), 321-333. MR 34 \#2613.

17. - Integral closure and $(x, y)^{n}=\left(x^{n}, y^{n}\right)$, Monatsh. Math. 71 (1967), 32-39. MR 34 \#4290.

18. R. Pendleton, A characterization of Q-domains, Bull. Amer. Math. Soc. 72 (1966), 499500. MR $32 \# 7578$.

19. P. Ribenboim, Anneaux normaux réels à caractère fini, Summa Brasil. Math. 3 (1956), 213-253. MR 20 \#3860. 
20. P. Roquette, On the prolongation of valuations, Trans. Amer. Math. Soc. 88 (1958), 42-56. MR 20 \#7019.

21. O. Zariski and P. Samuel, Commutative algebra, Vol. 1, University Series in Higher Math., Van Nostrand, Princeton, N. J., 1958. MR 19, 833.

22. —-, Commutative algebra, Vol. 2, University Series in Higher Math., Van Nostrand, Princeton, N. J., 1960. MR 22 \#11006.

Florida STATE UNIVERSity,

TAllahassee, Florida 32306 